\title{
Scalar leptoquarks at LHC
}

\section{Ilja Doršner*}

University of Split, Faculty of Electrical Engineering, Mechanical Engineering and Naval Architecture in Split (FESB)

Ruđera Boškovića 32, 21000 Split, Croatia

E-mail: dorsnerefesb.hr

The implications of large lepton-quark-leptoquark couplings for direct leptoquark searches at Large Hadron Collider (LHC) are addressed. It is shown that flavour physics constraints allow for sizeable couplings of leptoquarks to the Standard Model fermions if these interact exclusively with charged leptons and quarks of the same generation. The leptoquark production mechanisms at LHC are thoroughly discussed to demonstrate the importance of inclusion of a $t$-channel pair production and, in particular, a single leptoquark production if and when the leptoquark couplings are large. A recast of an existing CMS search at LHC for the second generation leptoquarks is accordingly presented. It is shown to yield the best direct limit to date on Yukawa coupling of a leptoquark that couples exclusively to a muon and a strange quark .

Proceedings of the Corfu Summer Institute 2015 "School and Workshops on Elementary Particle Physics and Gravity"

1-27 September 2015

Corfu, Greece

${ }^{*}$ Speaker. 
Leptoquarks (LQs) are hypothetical particles that emerge in a particularly natural fashion in extensions of the Standard Model (SM) that unify matter. They have one special property that sets them apart from all other elementary particles. Namely, LQs can turn quarks into leptons and vice versa [1]. They thus represent unique source of new physics that can be and has been extensively tested. LQ discovery would be a tentative signal of matter unification. This, on the other hand, would nicely dovetail with the observed unification of weak and electromagnetic interactions.

The leptoquark properties and potential experimental signals have been studied in great depth in literature. For a recent comprehensive review on the status of leptoquarks in precision experiments and at particle colliders see Ref. [2]. In this note I present a study of Ref. [3] that investigates a particular signal of a subset of scalar leptoquarks at LHC that is not in contradiction with the current experimental constraints. In particular, the study focuses on those leptoquark multiplets that do not contribute to nucleon decay at tree level.

All existing accelerator searches for light leptoquarks are assumption driven. Here I focus on one particular assumption one should find troublesome. Namely, it is commonly assumed that the pair production of leptoquarks is purely QCD driven. Note, however, that the sizeable Yukawa couplings of the leptoquarks with the SM fermions could influence pair production as is demonstrated later on. This regime would also make single leptoquark production very relevant at LHC [4].

The implications of large Yukawa couplings for a pair production and a single production of leptoquarks at LHC are investigated through a recast of an existing CMS search for the second generation leptoquark [5]. To make the study self-consistent it is shown that the current flavour constraints do not exclude parameter space where Yukawa couplings are large. The recast, on the other hand, shows that the current LHC data place more stringent limits on the scalar leptoquarks with large couplings to the SM fermions than what one infers from the flavour physics measurements.

The note is organised as follows. In Sec. 1 two scalar leptoquark multiplets that are relevant for the recast are presented and their couplings to the SM fermions are accordingly discussed. Leptoquark production mechanisms at LHC are discussed in Sec. 2. All relevant flavour physics constraints on the scalar leptoquark Yukawa couplings are provided in Sec. 3. The recast of the search for the second generation leptoquark is given in Sec. 4. This is followed with conclusions in Sec. 5.

\section{Framework}

There are only two scalar leptoquark multiplets - $(\mathbf{3}, 2,7 / 6)$ and $(\mathbf{3}, 2,1 / 6)$ - that are not dangerous for proton decay at tree level. (Leptoquarks are denoted via their transformation properties under the SM gauge group of $S U(3) \times S U(2) \times U(1)$. The normalisation is such that $Q=I_{3}+Y$, where $Q$ is the electric charge, $I_{3}$ stands for appropriate eigenvalue of the diagonal generator of $S U(2)$, and $Y$ represents the $U(1)$ (hyper)charge.) These two multiplets can thus have sizeable Yukawa couplings to matter and be light enough to be accessible in accelerator searches.

Let us, for definiteness, consider the SM extended with a single scalar LQ representation. This LQ, for aforementioned reasons, can be either $\tilde{R}_{2} \equiv(\mathbf{3}, \mathbf{2}, 1 / 6)$ or $R_{2} \equiv(\mathbf{3}, \mathbf{2}, 7 / 6)$. (Here, I use notation for the LQ states that was introduced in Ref. [6].) In fact, the study will be mainly concerned with a $Q=2 / 3$ component in $\tilde{R}_{2}$ and a $Q=5 / 3$ component in $R_{2}$. The LQ couplings of 
$\tilde{R}_{2}$ and $R_{2}$ to the SM fermions are briefly summarised next. A particular ansatz is also introduced to allow for a self-consistent recast of accelerator signatures.

\subsection{The $(3,2,1 / 6)$ case}

The only renormalizable term that describes interactions of $\tilde{R}_{2}$ with matter is given by

$$
\mathscr{L}_{\mathrm{Y}}=-y_{i j} \vec{d}_{R}^{i} \tilde{R}_{2}^{a} \varepsilon^{a b} L_{L}^{j, b}+\text { h.c. },
$$

where $i, j=1,2,3$ are flavour indices and $a, b=1,2$ are $S U(2)$ indices. $y_{i j}$ are elements of an arbitrary complex $3 \times 3$ Yukawa coupling matrix. After expanding $S U(2)$ indices, one gets

$$
\mathscr{L}_{\mathrm{Y}}=-y_{i j} \vec{d}_{R}^{i} e_{L}^{j} \tilde{R}_{2}^{2 / 3}+\left(y V_{\mathrm{PMNS}}\right)_{i j} \vec{d}_{R}^{i} v_{L}^{j} \tilde{R}_{2}^{-1 / 3}+\text { h.c. },
$$

where the LQ superscript denotes electric charge of a given $S U(2)$ doublet component and $V_{\mathrm{PMNS}}$ represents Pontecorvo-Maki-Nakagawa-Sakata mixing matrix. All fields in Eq. (1.2) are specified in the mass eigenstate basis.

The following ansatz is taken for Yukawa coupling matrix $y: y_{i j}=\delta_{i j} y_{i}=\delta_{i j} y_{j}, i, j=1,2,3$. $\tilde{R}_{2}^{2 / 3}$ thus couples exclusively to a charged lepton and a down-type quark of the same generation.

Decay width of $\tilde{R}_{2}^{2 / 3}$ to a particular decay channel is

$$
\Gamma\left(\tilde{R}_{2}^{2 / 3} \rightarrow d_{i} e_{i}^{+}\right)=\frac{m_{\mathrm{LQ}}}{16 \pi}\left|y_{i}\right|^{2},
$$

where $m_{\mathrm{LQ}}$ is the LQ mass. Correspondingly, branching ratios are given by

$$
\beta_{i}=\frac{\left|y_{i}\right|^{2}}{\left|y_{1}\right|^{2}+\left|y_{2}\right|^{2}+\left|y_{3}\right|^{2}}, \quad i=1,2,3 .
$$

These are the expressions that are used in numerical simulation.

\subsection{The $(3,2,7 / 6)$ case}

Yukawa couplings of $R_{2}$ to the SM fermions are

$$
\mathscr{L}_{\mathrm{Y}}=z_{i j} \bar{e}_{R}^{i} R_{2}^{a *} Q_{L}^{j, a}-y_{i j} \bar{u}_{R}^{i} R_{2}^{a} \varepsilon^{a b} L_{L}^{j, b}+\text { h.c.. }
$$

$y$ and $z$ in Eq. (1.5) are a priori arbitrary complex $3 \times 3$ Yukawa matrices. In the mass eigenstate basis one gets

$$
\mathscr{L}_{\mathrm{Y}}=z_{i j} \bar{e}_{R}^{i} d_{L}^{j} R_{2}^{2 / 3 *}+\left(z V_{\mathrm{CKM}}^{\dagger}\right)_{i j} \bar{e}_{R}^{i} u_{L}^{j} R_{2}^{5 / 3 *}+\left(y V_{\mathrm{PMNS}}\right)_{i j} \bar{u}_{R}^{i} v_{L}^{j} R_{2}^{2 / 3}-y_{i j} \bar{u}_{R}^{i} e_{L}^{j} R_{2}^{5 / 3}+\text { h.c. },
$$

where the LQ superscript denotes electric charge of a given $S U(2)$ doublet component and $V_{\mathrm{CKM}}$ represents Cabibbo-Kobayashi-Maskawa mixing matrix. Clearly, both components of $R_{2}$ have two sets of couplings to the SM fermions. These, on the other hand, are not connected via observed mixing matrices. This makes a self consistent analysis of accelerator signatures of $R_{2}$ rather difficult. In what follows a particular case of $R_{2}^{5 / 3}$ production at LHC when $y_{i j}=\delta_{i j} y_{i}=\delta_{i j} y_{j}$ and $z_{i j}=0, i, j=1,2,3$, is investigated. 


\section{Leptoquark production mechanisms at LHC}

The aim is to demonstrate that the leptoquark production at LHC is not driven solely by QCD induced pair production. It can be substantially influenced by the presence of relatively large Yukawa couplings of leptoquarks to the SM fermions. Moreover, if these couplings are taken to be large one also needs to take into consideration a single leptoquark production [4] and a $t$-channel leptoquark pair production.

A complete set of Feynman diagrams, at leading-order, that are relevant for a single leptoquark production of $R_{2}^{5 / 3}$ and $\tilde{R}_{2}^{2 / 3}$ at LHC is shown in Fig. 1 taking into account the ansatz for the couplings of these leptoquarks to the SM fermions. The diagrams shown in Fig. 1 are an $s$-channel (left panel) and a $t$-channel (right panel). Note the use of generic symbols for all the fields including the leptoquarks in Fig. 1. The study takes into account the composition of a proton and hence refers to $u(d$ and $s)$ to account for $R_{2}^{5 / 3}\left(\tilde{R}_{2}^{2 / 3}\right)$ production.
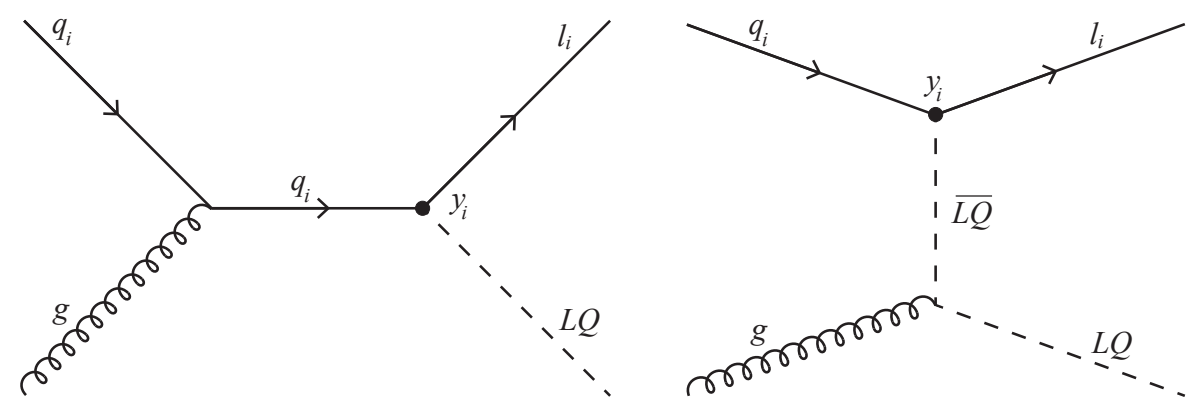

Figure 1: Complete set of the leading-order Feynman diagrams relevant for a single leptoquark production through an $s$-channel (left panel) and a $t$-channel (right panel) at LHC. Here, $y_{i}, i=1,2$, represents Yukawa coupling of a quark $q_{i}(u, d$ and $s)$ and a charged lepton $l_{i}(e$ and $\mu)$ with a leptoquark $(L Q)$.

Feynman diagrams that depict the LQ pair production are shown in Fig. 2. The QCD diagrams that contribute to a leptoquark pair production at LHC are numerous. Fig. 2 (left panel) features a representative diagram for gluon fusion. There is, on the other hand, only one type of the Yukawa coupling contribution to the leptoquark pair production and it corresponds to a $t$-channel process that is shown in Fig. 2 (right panel). The important point to notice is that the amplitude that corresponds to a $t$-channel is proportional to a square of absolute value of the relevant Yukawa coupling. This makes it especially relevant in the limit of large Yukawas.

The leptoquark pair production has three distinct regions. For small Yukawa couplings the total cross section is QCD driven. For intermediate Yukawas there exists a region with a negative interference between the QCD diagrams and the $t$-channel diagram, where the total cross section can be decreased by up to $15 \%$ depending on the quark type $(u, d$, and $s)$, the LQ mass $\left(m_{\mathrm{LQ}}\right)$ and the coupling strength $\left(y_{i}\right)$. Finally, there is a region of large Yukawas where the $t$-channel contribution not only dominates the QCD one but significantly enhances the total cross section.

In the following, the leading-order production cross section is calculated using MadGraph 5, v1.5.11 [7] after implementing the LQ models in FeynRules v1.6.16 [8] as introduced in Sec. 1. The calculations are performed for $\sqrt{s}=8 \mathrm{TeV}$ proton-proton center-of-mass energy using fixed renormalization $\left(\mu_{R}\right)$ and factorization $\left(\mu_{F}\right)$ scales set to $\mu_{R}=\mu_{F}=m_{\mathrm{LQ}} / 2$. The cross section for 

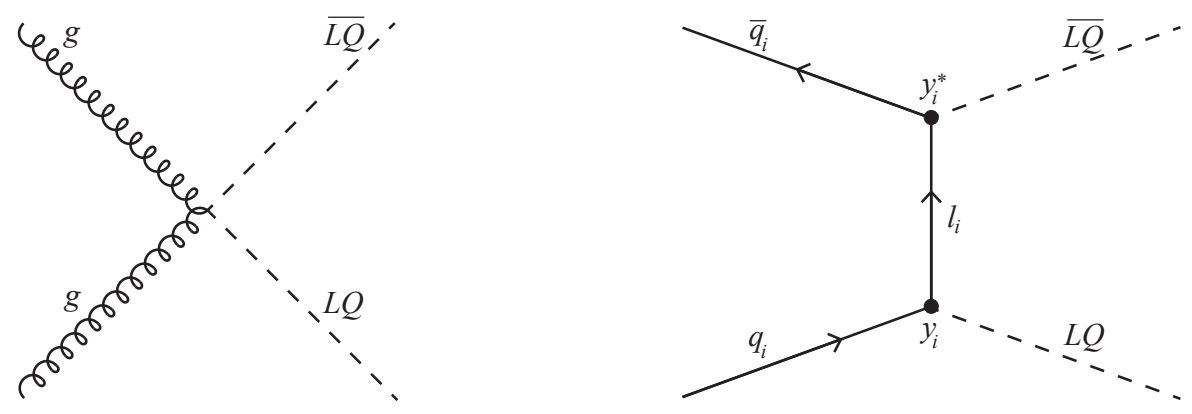

Figure 2: Feynman diagrams relevant for a pair production of leptoquarks at LHC. Representative diagram for a gluon fusion process is shown on the left. The diagram on the right represents a $t$-channel production mechanism. Here, $y_{i}, i=1,2$, represents Yukawa coupling of a quark $q_{i}(u, d$ and $s)$ and a charged lepton $l_{i}$ $(e$ and $\mu$ ) with a leptoquark $(L Q)$.

the single LQ production takes the following form,

$$
\sigma_{\text {single }}\left(y_{i}, m_{\mathrm{LQ}}\right)=a\left(m_{\mathrm{LQ}}\right)\left|y_{i}\right|^{2},
$$

where the coefficient $a\left(m_{\mathrm{LQ}}\right)$ depends on the leptoquark mass but not on its coupling to the SM fermions. Therefore, the cross section is calculated for $p p \rightarrow \overline{L Q} q_{i}$ together with $p p \rightarrow L Q \overline{q_{i}}$ for several $m_{\mathrm{LQ}}$ choices while setting the coupling $y_{i}$ to one, i.e, $y_{i}=1$. This produces the functional dependence $a\left(m_{\mathrm{LQ}}\right)$ through appropriate interpolation. Analogously, the cross section for the leptoquark pair production is assumed to take the form,

$$
\sigma_{\text {pair }}\left(y_{i}, m_{\mathrm{LQ}}\right)=a_{0}\left(m_{\mathrm{LQ}}\right)+a_{2}\left(m_{\mathrm{LQ}}\right)\left|y_{i}\right|^{2}+a_{4}\left(m_{\mathrm{LQ}}\right)\left|y_{i}\right|^{4},
$$

where the three terms correspond to the QCD pair production, an interference term, and a $t$-channel production, respectively. In order to solve for the coefficients, the cross section is calculated for a given $m_{\mathrm{LQ}}$ for three values of the $y_{i}$ couplings. This process is repeated at several mass points to produce functional dependence through appropriate interpolation.

The final results are shown in Fig. 3 in terms of contours of constant cross section in the $\left(m_{\mathrm{LQ}},\left|y_{i}\right|\right)$ plane. Fig. 3 contains separate predictions for $y_{u e}, y_{d e}$ and $y_{s \mu}$ couplings. $y_{1}=y_{u e}$ stands for Yukawa coupling of $R_{2}^{5 / 3}$ with an electron and an up-quark. In case of $\tilde{R}_{2}^{2 / 3}$ one needs to treat separately contributions from $y_{1}=y_{d e}$ and $y_{2}=y_{s \mu}$. The contours of constant cross section in Fig. 3 for the single leptoquark production are shown in solid lines, while the contours for the leptoquark pair production are shown in dotted lines.

The leptoquark pair production is clearly fixed by the QCD pair production for small Yukawa couplings. This behavior corresponds to a region where dotted lines run vertically in Fig. 3. However, in the large coupling regime, the total cross section can be significantly enhanced by the $t$-channel contribution. The leptoquark single production, on the other hand, drops less rapidly with larger LQ masses compared to the LQ pair production. In other words, the contributions from this production mechanism become increasingly important at larger LQ masses. The relative strengths of two cross sections depend on the parton distribution functions of the initial state partons. Namely, the largest (smallest) effect is seen for the $y_{u e}\left(y_{s \mu}\right)$ case.

To sum up, the contributions from the additional production mechanisms have dramatic impact on the leptoquark phenomenology at the LHC. It is shown that this also holds at the level of 

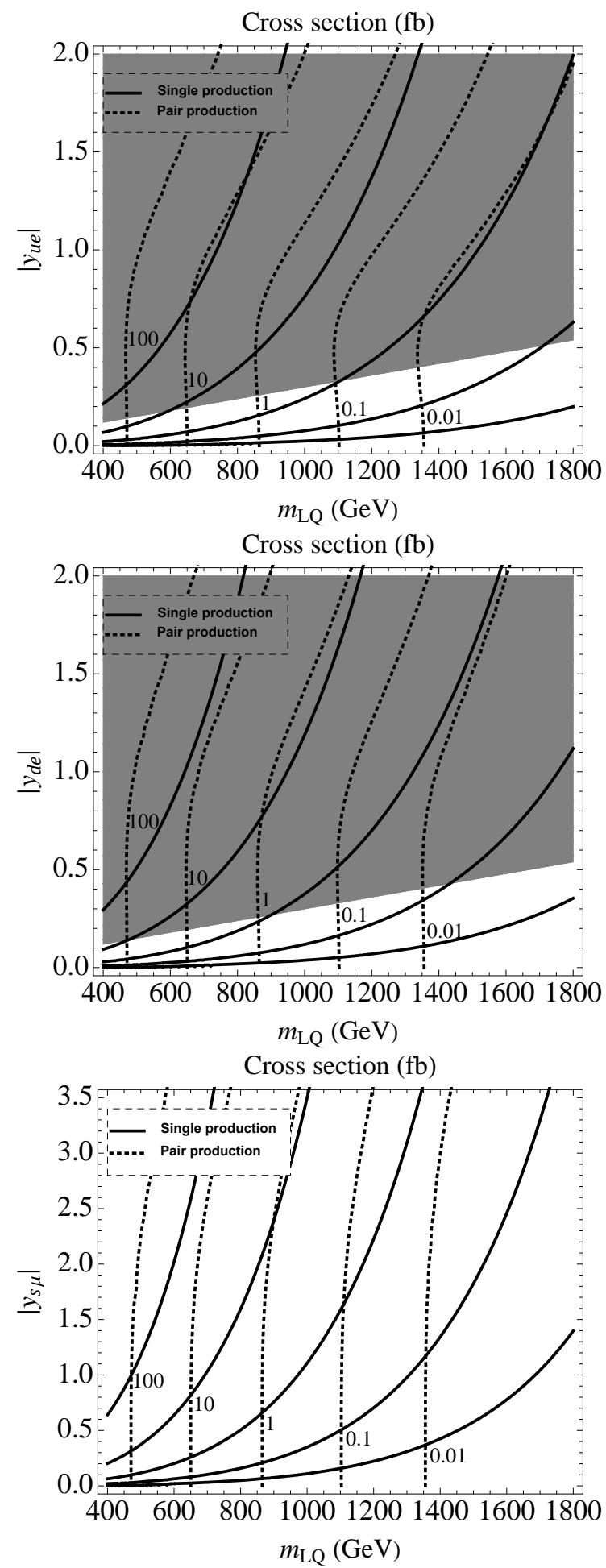

Figure 3: Contours of constant leading-order cross sections for single leptoquark production (solid lines) and the leptoquark pair production (dotted lines) at $\sqrt{s}=8 \mathrm{TeV}$ center-of-mass energy in proton-proton collisions in the $\left(m_{\mathrm{LQ}},\left|y_{i}\right|\right)$ planes. The region shown in grey is excluded from the atomic parity violation constraints. 
analysis in Sec. 4. Interestingly enough, the recast of the existing CMS search for second generation leptoquarks yields an improved constraint on the LQ parameter space. Such analysis would be even more important for the first generation leptoquarks in view of preceding discussion.

Before the recast is presented, it remains to be seen whether large Yukawa couplings are allowed by existing flavour physics measurements. This issue is discussed in the next section.

\section{Flavour constraints}

The study I discuss pursues a simplified scenarios where only one entry on the diagonal of $y$ for either $\tilde{R}_{2}^{2 / 3}$ or $R_{2}^{5 / 3}$ dominates. I now show that this ansatz is consistent with constraints from the flavour physics experiments. This also turns out to be the only viable scenario whenever one investigates regime of large Yukawa couplings for $\tilde{R}_{2}^{2 / 3}$ leptoquark.

The leptoquark production mechanism effects are not affected by $y_{3}$ coupling at all due to the particularities of the proton composition. I thus investigate constraints on $\left|y_{1}\right|,\left|y_{2}\right|$ and $\left|y_{1} y_{2}^{*}\right|$ for both $\tilde{R}_{2}^{2 / 3}$ and $R_{2}^{5 / 3}$ as a function of the LQ mass $m_{\mathrm{LQ}}$. I refer to $y_{1}$ and $y_{2}$ of $\tilde{R}_{2}^{2 / 3}\left(R_{2}^{5 / 3}\right)$ as $y_{d e}$ and $y_{s \mu}\left(y_{u e}\right.$ and $\left.y_{c \mu}\right)$, respectively.

A meaningful upper bound on $\left|y_{1}\right| / m_{\mathrm{LQ}}$ can be inferred from atomic parity violation (APV) experiments, whereas a tight constraint on $\left|y_{d e} y_{s \mu}^{*}\right| / m_{\mathrm{LQ}}^{2}$ arises from $K_{L} \rightarrow \mu^{-} e^{+}$process. A main constraint on $\left|y_{u e} y_{c \mu}^{*}\right| / m_{\mathrm{LQ}}^{2}$ that turns out to be rather weak originates from $D^{0} \rightarrow \mu^{-} e^{+}$. The bound on $\left|y_{s \mu}\right|$ due to experimental data from flavour physics including $g-2$ of muon is also weak. $\left|y_{c \mu}\right|$ coupling of $R_{2}$ is only slightly constrained by data on $g-2$ of muon and it can be of the order of unity for experimentally viable LQ masses. I discuss these constraints in what follows in more detail.

\subsection{Atomic parity violation (APV)}

The effective Lagrangian that leads to APV can be written as [9]

$$
\mathscr{L}_{\mathrm{PV}}=\frac{G_{F}}{\sqrt{2}} \sum_{q=u, d}\left(C_{1 q} \bar{e} \gamma^{\mu} \gamma_{5} e \bar{q} \gamma_{\mu} q+C_{2 q} \bar{e} \gamma^{\mu} e \bar{q} \gamma_{\mu} \gamma_{5} q\right)
$$

The SM coefficients due to the $Z$ boson exchange are $C_{1 u}^{\mathrm{SM}}=-1 / 2+4 / 3 \sin ^{2} \theta_{W}$ and $C_{1 d}^{\mathrm{SM}}=$ $1 / 2-2 / 3 \sin ^{2} \theta_{W}$. The higher-order corrections within the SM are determined in Refs. [10, 11] enabling one to generate very precise constraints on the potential contributions from new physics. APV is dependent on the nuclear weak charge $Q_{W}(Z, N)=-2\left[(2 Z+N) C_{1 u}+(2 N+Z) C_{1 d}\right]$ [9], where $C_{1 u}=C_{1 u}^{\mathrm{SM}}+\delta C_{1 u}$ and $C_{1 d}=C_{1 d}^{\mathrm{SM}}+\delta C_{1 d} . \delta C_{1 u}\left(\delta C_{1 u}\right)$ is the new physics contribution generated by the presence of $R_{2}^{5 / 3}\left(\tilde{R}_{2}^{2 / 3}\right.$ and/or $\left.R_{2}^{2 / 3}\right)$. $Z$ in $Q_{W}(Z, N)$ represents atomic number and $N$ stands for neutron number.

The experimentally extracted value $Q_{W}(\mathrm{Cs})=-73.20(35)$ for cesium atom $\left({ }^{133} \mathrm{Cs}\right)[12,13]$ is in very good agreement with the SM result $Q_{W}(\mathrm{Cs})=-73.15(35)$ [14]. This yields tight constraints on effective coefficients $\delta C_{1 u}$ and $\delta C_{1 d}$ that, for the LQ contribution, read

$$
\delta C_{1 u(d)}=\frac{\sqrt{2}}{G_{F}} \frac{\left|y_{u(d) e}\right|^{2}}{8 m_{\mathrm{LQ}}^{2}} .
$$


These translate into the following limits on $\left|y_{d e}\right|$ and $\left|y_{u e}\right|$ if one requires a $2 \sigma$ agreement with the experimental measurement of $Q_{W}(\mathrm{Cs})$ :

$$
\left|y_{d e}\right| \leq 0.34\left(\frac{m_{\mathrm{LQ}}}{1 \mathrm{TeV}}\right), \quad\left|y_{\text {ue }}\right| \leq 0.36\left(\frac{m_{\mathrm{LQ}}}{1 \mathrm{TeV}}\right) .
$$

These bounds are extracted under the assumption that only one of the two LQ contributions is present at a given moment. This assumption cannot be realised if one considers $R_{2}$ leptoquark and takes $z_{11} \neq 0$. It is, however, not possible to cancel $\delta C_{1 u}$ against $\delta C_{1 d}$ or vice versa. The upper bounds presented in Eq. (3.3) are thus applicable in the most general case. I accordingly use these bounds to exclude shaded regions in two upper panels in Fig 3.

3.2 $K_{L} \rightarrow \mu^{-} e^{+}$

The diagonal couplings of $\tilde{R}_{2}^{2 / 3}$ enter at the tree level into the lepton flavour violating $K_{L} \rightarrow$ $\mu^{-} e^{+}$decay amplitude. The decay width reads [15]

$$
\Gamma_{K_{L} \rightarrow \mu^{-} e^{+}}=\frac{\left|y_{s \mu} y_{d e}^{*}\right|^{2}}{512 \pi} \frac{m_{K}^{3} f_{K}^{2}}{m_{\mathrm{LQ}}^{4}}\left(\frac{m_{\mu}}{m_{K}}\right)^{2}\left[1-\left(\frac{m_{\mu}}{m_{K}}\right)^{2}\right]^{2} .
$$

Using lattice QCD result $f_{K}=156.1(0.8) \mathrm{MeV}$ [16] and $B R\left(K_{L} \rightarrow \mu^{ \pm} e^{\mp}\right)<4.7 \times 10^{-12}$ [17], the relevant bound reads

$$
\left|y_{s \mu} y_{d e}^{*}\right|<2.1 \times 10^{-5}\left(\frac{m_{\mathrm{LQ}}}{1 \mathrm{TeV}}\right)^{2} \text {. }
$$

3.3 $D^{0} \rightarrow \mu^{-} e^{+}$

The diagonal couplings of $R_{2}^{5 / 3}$ to the SM fermions enter at the tree level into the lepton flavour violating $D^{0} \rightarrow \mu^{-} e^{+}$decay amplitude. The decay width reads

$$
\Gamma_{D^{0} \rightarrow \mu^{+} e^{-}}=\frac{\left|y_{c \mu} y_{u e}^{*}\right|^{2}}{256 \pi} \frac{m_{D}^{3} f_{D}^{2}}{m_{\mathrm{LQ}}^{4}}\left(\frac{m_{\mu}}{m_{D}}\right)^{2}\left[1-\left(\frac{m_{\mu}}{m_{D}}\right)^{2}\right]^{2} .
$$

Using lattice QCD result $f_{D}=209.2 \mathrm{MeV}$ [18] and taking $B R\left(D^{0} \rightarrow \mu^{ \pm} e^{\mp}\right)<2.6 \times 10^{-7}$ [17], the bound is

$$
\left|y_{c \mu} y_{u e}^{*}\right|<0.6\left(\frac{m_{\mathrm{LQ}}}{1 \mathrm{TeV}}\right)^{2} \text {. }
$$

\section{$3.4 g-2$ of muon}

The $\tilde{R}_{2}^{2 / 3}$ coupling $y_{s \mu}$ contributes to the muon $g-2$ with a leptoquark and a strange quark within the loop. However, following Refs. [19, 20, 15], it is easy to show that the muon $g-2$ anomaly does not constrain $y_{s \mu}$ at all. This is due to a smallness of the strange quark mass and a substantial cancellations of two relevant contributions that enter into a shift of the muon anomalous moment with respect to the SM value. The electric charge of $R_{2}^{5 / 3}$ component, on the other hand, does not allow for the aforementioned cancellation. The consequence of that is a mild constraint on $\left|y_{c \mu}\right| / m_{\mathrm{LQ}}$ that reads [20]

$$
\left|y_{c \mu}\right| \leq 1.0\left(\frac{m_{\mathrm{LQ}}}{1 \mathrm{TeV}}\right)
$$




\begin{tabular}{c|ccccc}
$m_{\mathrm{LQ}}(\mathrm{GeV})$ & 500 & 700 & 900 & 950 & $\geq 1000$ \\
\hline$S_{T}>(\mathrm{GeV})$ & 685 & 935 & 1135 & 1175 & 1210 \\
$M_{\mu \mu}>(\mathrm{GeV})$ & 150 & 195 & 230 & 235 & 245 \\
$M_{\min }(\mu, j)>(\mathrm{GeV})$ & 155 & 295 & 535 & 610 & 690 \\
\hline \hline Signal yield $<$ at $95 \% \mathrm{CL}$ & 34 & 9.8 & 5.6 & 3.5 & 1.8
\end{tabular}

Table 1: Final selection cuts as used by the CMS collaboration. The cuts are optimized for the QCD pair production and depend on the LQ mass hypothesis. The last row shows the observed 95\% C.L. upper limit on the allowed signal yield after the final selection cuts are applied [5].

The preceding discussion demonstrates that if one takes $y_{s \mu}$ to be large, i.e., an order one quantity, then $y_{d e}$ has to be very small in order to satisfy Eq. (3.5). (The situation with the $R_{2}^{5 / 3}$ couplings is somewhat more involved. The flavour physics constraints allow for $y_{u e}$ and $y_{c \mu}$ to simultaneously be of relatively large value.) This means that one can neglect $y_{d e}$ altogether if one is to study the effects of large $y_{s \mu}$ at LHC. The recast of the second generation leptoquark search by the CMS collaboration I present next is thus self-consistent and well justified in that regime.

\section{Recasting the CMS search for the second generation leptoquarks}

The CMS collaboration has recently reported a search for the second generation scalar LQs based on $19.6 \mathrm{fb}^{-1}$ of data at $\sqrt{s}=8 \mathrm{TeV}$ proton-proton center-of-mass energy [5]. The CMS analysis assumption is that the $\mu-L Q-s$ coupling $y_{s \mu}$ is small. The LQ pair production is thus completely fixed by QCD. This assumption is relaxed in the recast and the impact of large $y_{s \mu}$ on the existing experimental search is studied. In particular, large coupling leads to substantial signal yield from $t$-channel leptoquark pair production as well as single leptoquark production. Consequently, the recast of the CMS search reported in [5] sets an improved limit on the second generation scalar leptoquark parameter space.

Here I outline the details of the recast [3]. FeynRules v1.6.16 [8] was used to implement the model containing $(\mathbf{3}, \mathbf{2}, 1 / 6)$ scalar representation and the interactions defined by the Lagrangian in Eq. (1.2). MadGraph 5 v1.5.11 [7] was used to generate $p p \rightarrow \tilde{R}_{2}^{2 / 3} \tilde{R}_{2}^{2 / 3 *}, p p \rightarrow \tilde{R}_{2}^{2 / 3 *} \mu^{+}$, and $p p \rightarrow \tilde{R}_{2}^{2 / 3} \mu^{-}$processes, followed by the LQ decays to a muon and a strange quark. The decay branching ratio was taken to be $\beta_{2}=1$, which is in line with the assumption of a single large coupling and is consistent with the flavour physics constraints presented in Sec. 3. The analysis presented here is based on the leading-order calculations. The inferred exclusion limits are thus very conservative. In order to partially account for large corrections, the factorization and renormalization scales were fixed to $\mu_{F}=\mu_{R}=m_{\mathrm{LQ}} / 2$. Showering and hadronization effects were simulated using Pythia v6.426 [21]. For the detector simulation the default implementation of the CMS detector in Delphes v3.0.9 [22] was used. In addition, the default implementation was modified by switching to the anti- $k_{T}$ jet algorithm with distance parameter $R=0.5$, and by changing the muon isolation criteria in accordance with [5].

The preselection cuts were adopted from Ref. [5]. The final selection cuts, on the other hand, were applied on the following three variables: (i) the invariant mass of the dimuon pair $\left(M_{\mu \mu}\right)$, (ii) the scalar sum of the transverse momenta of the two leading $p_{T}$ muons and the two leading $p_{T}$ jets 

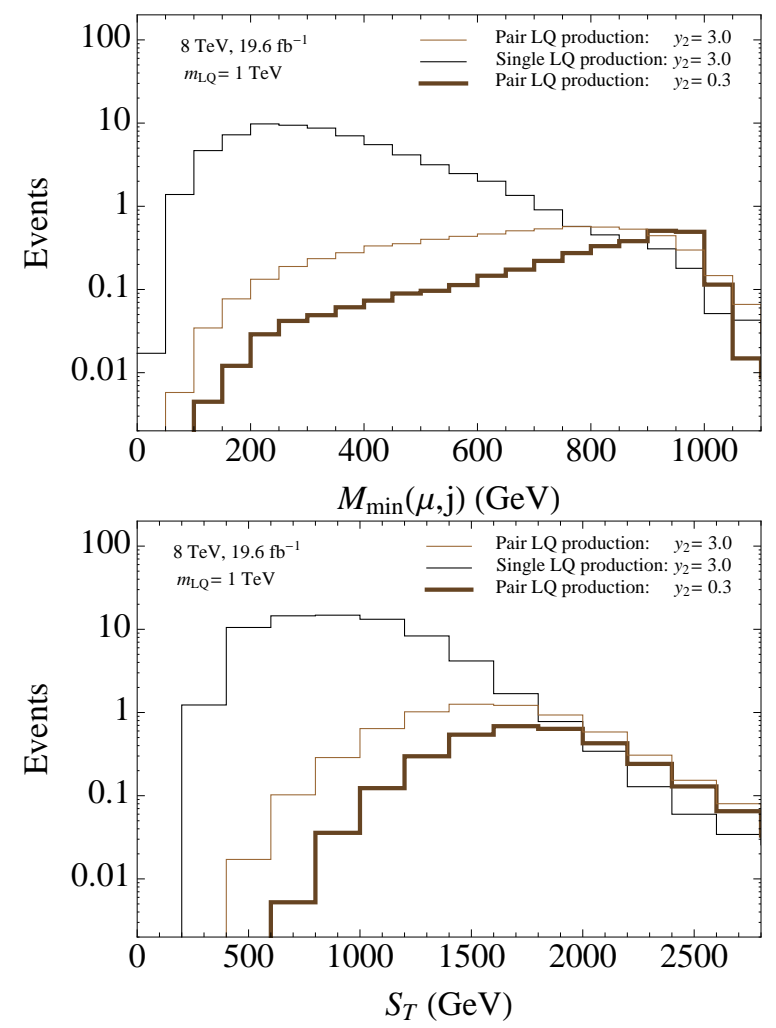

Figure 4: The signal event distributions in $M_{\min }(\mu, j)$ and $S_{T}$ variables after application of preselection cuts. The predictions for single LQ production for $y_{2}=3$ are shown with black thin lines, while the predictions for pair production with the same value of the coupling are shown with light brown thin lines. The predictions due to the QCD pair production are shown with dark brown thick lines. The LQ mass is taken to be $m_{\mathrm{LQ}}=$ $1 \mathrm{TeV}$.

$\left(S_{T}\right)$, and (iii) the smallest of the two muon-jet invariant masses that minimizes the leptoquark mass difference $\left(M_{\min }(\mu, j)\right)$. The final cuts used by the CMS collaboration are reported in Table 1 . In order to illustrate the impact of the final selection cuts in the large coupling regime I present in Fig. 4 the signal event distributions in $S_{T}$ and $M_{\min }(\mu, j)$ variables after the application of preselection cuts. Here, I choose LQ mass to be $m_{\mathrm{LQ}}=1 \mathrm{TeV}$ which is close to a present exclusion limit. The signal yield from the QCD pair production is shown with dark-brown thick line. The distributions in $S_{T}\left(M_{\min }(\mu, j)\right)$ variable tend to peak for $S_{T} \sim 2 m_{\mathrm{LQ}}\left(M_{\min }(\mu, j) \sim m_{\mathrm{LQ}}\right)$. The contribution due to LQ pair production for the value of the coupling set to $y_{2}=3.0$ is shown with light-brow thin lines. The integrated signal yield is larger when compared to the previous case due to additional contributions from the $t$-channel diagrams. I also show the contributions from a single leptoquark production for the coupling $y_{2}=3.0$ with black thin lines. While the integrated signal yield is significantly larger with respect to previous cases, the events tend to have considerably smaller $S_{T}$ and $M_{\min }(\mu, j)$ values. $M_{\min }(\mu, j)$ is clearly efficient only when it is a leptoquark pair that decays into the final state particles. The muon coming from the production tends to have considerable smaller $p_{T}$ with respect to the muon coming from the leptoquark decay. The same holds for the second leading $p_{T}$ jet that most likely originates from the real QCD radiation as compared with the leading $p_{T}$ jet that most likely comes from the LQ decay. This explains the softer distributions in 


\begin{tabular}{c|cccc}
$m_{\mathrm{LQ}}(\mathrm{GeV})$ & $y_{2}=0.3$ & $y_{2}=1.0$ & $y_{2}=2.0$ & $y_{2}=3.0$ \\
\hline 500 & $\mathbf{6 0 0}+\mathbf{8 . 2}$ & $\mathbf{6 0 0}+\mathbf{8 9}$ & $\mathbf{7 2 0}+\mathbf{3 3 0}$ & $\mathbf{1 3 0 0}+\mathbf{7 0 0}$ \\
700 & $\mathbf{5 5}+\mathbf{0 . 9 8}$ & $\mathbf{5 6}+\mathbf{1 1}$ & $\mathbf{6 4}+\mathbf{4 1}$ & $\mathbf{1 1 0}+\mathbf{8 1}$ \\
900 & $\mathbf{6 . 5}+\mathbf{0 . 1 0}$ & $\mathbf{6 . 5}+\mathbf{1 . 2}$ & $\mathbf{7 . 0}+\mathbf{4 . 5}$ & $\mathbf{1 1}+\mathbf{8 . 4}$ \\
1000 & $\mathbf{2 . 2}+\mathbf{0 . 0 3}$ & $\mathbf{2 . 2}+\mathbf{0 . 3 3}$ & $\mathbf{2 . 3}+\mathbf{1 . 1}$ & $\mathbf{3 . 1}+\mathbf{2 . 3}$ \\
1050 & $1.5+0.02$ & $\mathbf{1 . 5}+\mathbf{0 . 2 7}$ & $\mathbf{1 . 5}+\mathbf{1 . 0}$ & $\mathbf{2 . 1}+\mathbf{2 . 1}$ \\
1100 & $0.96+0.02$ & $0.96+0.21$ & $\mathbf{1 . 0}+\mathbf{0 . 8 2}$ & $\mathbf{1 . 4}+\mathbf{1 . 6}$ \\
1150 & $0.62+0.02$ & $0.62+0.17$ & $0.66+0.75$ & $\mathbf{0 . 9 2}+\mathbf{1 . 4}$ \\
1200 & $0.41+0.01$ & $0.41+0.14$ & $0.44+0.55$ & $\mathbf{0 . 6 0}+\mathbf{1 . 3}$ \\
1300 & $0.17+0.01$ & $0.17+0.09$ & $0.19+0.37$ & $0.26+0.74$ \\
1400 & $0.07+0.00$ & $0.07+0.06$ & $0.08+0.24$ & $0.12+0.52$
\end{tabular}

Table 2: The simulated signal yields after the application of final selection cuts. The predictions are shown as the sum of the pair production and the single production contributions $N_{\text {evs }}$ (Pair production) + $N_{\text {evs }}$ (Single production). The points in parameter space spanned by $y_{2} \equiv y_{s \mu}$ and $m_{\mathrm{LQ}}$ that are shown in bold are excluded by the existing data.

the $S_{T}$ variable for the single LQ production. Conclusively, the majority of the signal is lost if one applies the final selection cuts from Table 1. The recast nevertheless keeps the CMS collaboration cuts in order to rely on the official background predictions.

I present the results of the recast in Table 2. Table 2 contains signal event yields for certain choices of coupling $y_{2} \equiv y_{s \mu}$ and $m_{\mathrm{LQ}}$. The predictions are given as the sum of two numbers representing the individual contributions from the LQ pair and single production, respectively.

The predictions for LQ pair production for small couplings agree well with the results reported in Table 4 of [5] after using the next-to-leading order QCD corrected cross sections. This serves as an important cross-check of the simulation procedure. The main observation at this point is that, for large values of the coupling $y_{2}$, the contributions from the $t$-channel pair production and, particularly, single LQ production become important. Moreover, the latter process is especially relevant for larger LQ masses due to the phase space suppression in pair production. The point made here gains on importance as the recast sets stronger exclusion limits on LQ parameter space.

The predictions for signal yields from Table 2 can be translated into the exclusions in $\left(m_{\mathrm{LQ}}, y_{s \mu}\right)$ plane. Here the recast relies on the official statistical analysis performed by the CMS collaboration. The observed 95\% C.L. upper limit on the allowed signal yields after the application of final selection cuts is shown in the last row of Table 1 . These limits are obtained by rescaling the observed 95\% C.L. upper limits on the production cross sections as reported in Fig. 8 of [5]. The rescaling factors are the signal event yields reported in Table 4 of Ref. [5] divided by the SM cross section from Table 1 of Ref. [5].

The improved constraints on the second generation leptoquarks are shown in Fig. 5. The parameter region in $\left(m_{\mathrm{LQ}}, y_{s \mu}\right)$ plane that is excluded at $95 \%$ C.L. by the existing LHC data is shown in grey. The signal yield as a function of the coupling and mass, i.e., $N_{\mathrm{evs}}\left(m_{\mathrm{LQ}}, y_{s \mu}\right)$, is obtained after interpolating over the points shown in Table 2. The excluded region corresponds to the points for which $N_{\mathrm{evs}}\left(m_{\mathrm{LQ}}, y_{s \mu}\right)$ is greater than the appropriate value reported in Table 1 . As advocated before, the limits on the LQ masses are more stringent for larger values of $\mu-L Q_{-}$ 
$s$ coupling. (After this recast was completed the CMS collaboration has performed the analysis of the LQ search through the single production mechanism for both first and second generation leptoquarks [23]. The results of the CMS analysis corroborate claims advocated in the recast [3].)

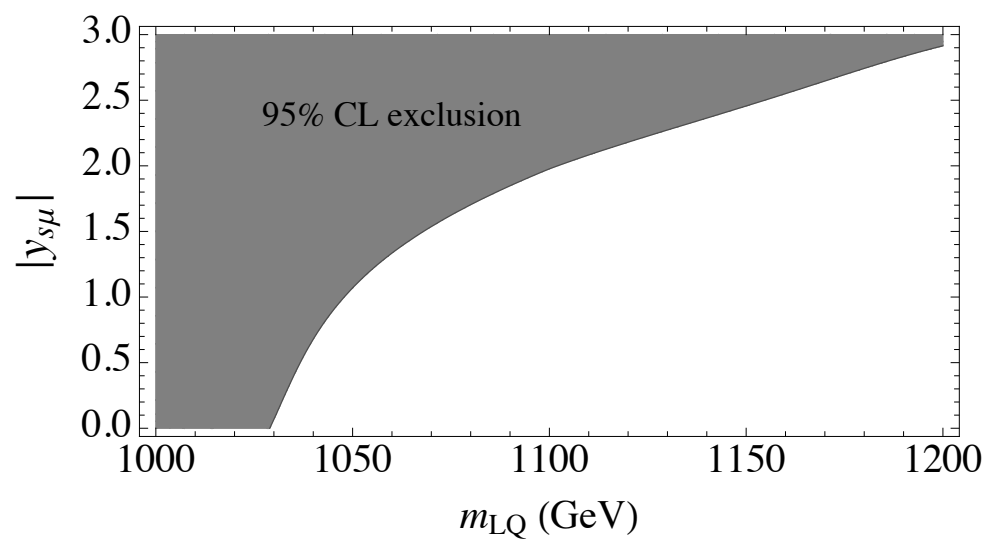

Figure 5: Direct constrains on the parameter space of the second generation scalar leptoquarks by the existing LHC data. The region shown in grey is excluded at 95\% C.L..

\section{Conclusions}

A study of a pair production and a single production of leptoquarks in the regime when leptoquarks couple strongly to a charged lepton and a quark of the same generation is presented. This is accomplished for two leptoquark multiplets that do not cause proton decay at tree level. The most stringent flavour constraints on the strength of the relevant Yukawa couplings are analysed to demonstrate viable of the large Yukawa regime.

The importance of inclusion of the single leptoquark production and the $t$-channel pair production is demonstrated through a recast of an existing CMS search at LHC for the second generation leptoquark that couples to a muon and a strange quark. The recast yields the best limit on the Yukawa coupling and mass of the second generation leptoquark to date.

\section{Acknowledgments}

I.D. thanks S. Fajfer and A. Greljo for inspiring discussion and collaboration with regard to this project. This work has been supported in part by Croatian Science Foundation under the project 7118.

\section{References}

[1] J. C. Pati and A. Salam, Phys. Rev. D 10 (1974) 275 Erratum: [Phys. Rev. D 11 (1975) 703]. doi:10.1103/PhysRevD.10.275, 10.1103/PhysRevD.11.703.2

[2] I. Doršner, S. Fajfer, A. Greljo, J. F. Kamenik and N. Košnik, arXiv:1603.04993 [hep-ph]. 
[3] I. Doršner, S. Fajfer and A. Greljo, JHEP 1410 (2014) 154 doi:10.1007/JHEP10(2014)154 [arXiv:1406.4831 [hep-ph]].

[4] A. Belyaev, C. Leroy, R. Mehdiyev and A. Pukhov, JHEP 0509 (2005) 005 doi:10.1088/1126-6708/2005/09/005 [hep-ph/0502067].

[5] [CMS Collaboration], CMS-PAS-EXO-12-042.

[6] W. Buchmuller, R. Ruckl and D. Wyler, Phys. Lett. B 191 (1987) 442 Erratum: [Phys. Lett. B 448 (1999) 320]. doi:10.1016/0370-2693(87)90637-X

[7] J. Alwall, M. Herquet, F. Maltoni, O. Mattelaer and T. Stelzer, JHEP 1106 (2011) 128 doi:10.1007/JHEP06(2011)128 [arXiv:1106.0522 [hep-ph]].

[8] N. D. Christensen and C. Duhr, Comput. Phys. Commun. 180 (2009) 1614 doi:10.1016/j.cpc.2009.02.018 [arXiv:0806.4194 [hep-ph]].

[9] M. I. Gresham, I. W. Kim, S. Tulin and K. M. Zurek, Phys. Rev. D 86 (2012) 034029 doi:10.1103/PhysRevD.86.034029 [arXiv:1203.1320 [hep-ph]].

[10] W. J. Marciano and A. Sirlin, Phys. Rev. D 27 (1983) 552. doi:10.1103/PhysRevD.27.552

[11] M. J. Ramsey-Musolf, Phys. Rev. C 60 (1999) 015501 doi:10.1103/PhysRevC.60.015501 [hep-ph/9903264].

[12] C. S. Wood, S. C. Bennett, D. Cho, B. P. Masterson, J. L. Roberts, C. E. Tanner and C. E. Wieman, Science 275 (1997) 1759. doi:10.1126/science.275.5307.1759

[13] J. Guena, M. Lintz and M. A. Bouchiat, Phys. Rev. A 71 (2005) 042108 doi:10.1103/PhysRevA.71.042108 [physics/0412017 [physics.atom-ph]].

[14] S. G. Porsev, K. Beloy and A. Derevianko, Phys. Rev. Lett. 102 (2009) 181601 doi:10.1103/PhysRevLett.102.181601 [arXiv:0902.0335 [hep-ph]].

[15] I. Doršner, J. Drobnak, S. Fajfer, J. F. Kamenik and N. Košnik, JHEP 1111 (2011) 002 doi:10.1007/JHEP11(2011)002 [arXiv:1107.5393 [hep-ph]].

[16] S. Aoki et al., Eur. Phys. J. C 74 (2014) 2890 doi:10.1140/epjc/s10052-014-2890-7 [arXiv:1310.8555 [hep-lat]].

[17] J. Beringer et al. [Particle Data Group Collaboration], Phys. Rev. D 86 (2012) 010001. doi:10.1103/PhysRevD.86.010001

[18] H. Na, C. T. H. Davies, E. Follana, G. P. Lepage and J. Shigemitsu, Phys. Rev. D 86 (2012) 054510 doi:10.1103/PhysRevD.86.054510 [arXiv:1206.4936 [hep-lat]].

[19] K. m. Cheung, Phys. Rev. D 64 (2001) 033001 doi:10.1103/PhysRevD.64.033001 [hep-ph/0102238].

[20] I. Doršner, S. Fajfer, N. Košnik and I. Nišandžić, JHEP 1311 (2013) 084 doi:10.1007/JHEP11(2013)084 [arXiv:1306.6493 [hep-ph]].

[21] T. Sjostrand, S. Mrenna and P. Z. Skands, JHEP 0605 (2006) 026 doi:10.1088/1126-6708/2006/05/026 [hep-ph/0603175].

[22] J. de Favereau et al. [DELPHES 3 Collaboration], JHEP 1402 (2014) 057 doi:10.1007/JHEP02(2014)057 [arXiv:1307.6346 [hep-ex]].

[23] V. Khachatryan et al. [CMS Collaboration], Phys. Rev. D 93 (2016) no.3, 032005 doi:10.1103/PhysRevD.93.032005 [arXiv:1509.03750 [hep-ex]]. 